\title{
On the Green Functions of 1-Dimensional Diffusion Processes
}

\author{
By
}

Yuji Kasahara, Shin'ichi Kotani and Hisao Watanabe

\section{§0. Introduction}

Let $X=\left\{X_{t}\right\}$ be a Markov process with a measurable state $S$. The Green operator $G_{\alpha}(\alpha \geqq 0)$ of $X$ is usually defined by

$$
\begin{aligned}
G_{\omega} f(x) & =E_{x}\left[\int_{0}^{\infty} e^{-\alpha t} f\left(X_{t}\right) d t\right] \\
& =\int_{S} G_{\alpha}(x, d y) f(y)
\end{aligned}
$$

for every bounded measurable function $f(x)$, where

$$
G_{a}(x, E)=\int_{0}^{\infty} e^{-\infty t} P_{x}\left(X_{t} \in E\right) d t .
$$

For every $\alpha>0, G_{\alpha}$ is always well-defined, but $G_{0}$ does not exist in general, as is seen in the case of 1- or 2-dimensional Brownian motions. However, in many cases, $G_{0} f$ can be defined to be the limit of $G_{a} f$ as $\alpha \rightarrow 0$ if we restrict $f$ to be in a certain class of functions, called the domain of the potential operator. There have been a lot of works on potential operators for Markov processes, e.g. G. Hunt [1], J. Takeuch,i T. Yamada and S. Watanabe [2], G. Kemeny-J.L. Snell [3], R. Kondo [4], K. Yosida [5], K. Sato [6] and T. Arakawa and J. Takeuchi [7]. An important class of recurrent Markov processes for which we can define potential operators is the following: Suppose that $G_{w}(x, d y)(\alpha>0)$ has density $g_{a}(x, y)$ with respect to a measure $\nu(d y)$ of the following form:

$$
g_{\alpha}(x, y)=h(\alpha)+u(x, y)+\varepsilon(x, y ; \alpha),
$$

where $h(\alpha) \rightarrow \infty$ and $\varepsilon(x, y ; \alpha) \rightarrow 0$ as $\alpha \rightarrow 0$. Then it is clear that for a reasonably broad class of functions with $\nu$-integral 0 , i.e. $\int f(x) \nu(d x)=0$, we have

Communicated by K. Itô, March 30, 1978.

* Department of Mathematics, Kyoto University.

** Department of Applied Science, Faculty of Engineering, Kyushu University. 


$$
\begin{aligned}
\lim _{\alpha \rightarrow 0} G_{a s} f(x) & =\lim _{\alpha \rightarrow 0} \int_{S} g_{\alpha}(x, y) f(y) \nu(d y) \\
& =\lim _{\alpha \rightarrow 0} \int_{s}\left\{g_{\alpha s}(x, y)-h(\alpha)\right\} f(y) \nu(d y) \\
& =\int_{S} u(x, y) f(y) \nu(d y),
\end{aligned}
$$

which suggests that we can define $G_{0}$ as

$$
G_{0} f(x)=\int_{S} u(x, y) f(y) \nu(d y) .
$$

For example, in the case of 1-dimensional Brownian motion, we have

$$
\nu(d y)=d y, \quad h(\alpha)=\frac{1}{\sqrt{2 \alpha}}, \quad u(x, y)=-|x-y|,
$$

and

$$
G_{0} f(x)=\int_{-\infty}^{\infty}|x-y| f(y) \nu(d y)
$$

for every function $f$ with compact support satisfying $\int_{-\infty}^{\infty} f(x) \nu(d x)=0$. We remark that the representation $(0.1)$ is explicitly given for stable processes [2] and Bessel processes [7]. This representation is useful in many cases; for example, one of the authors [8] has recently obtained a limit theorem for occupation times based on the representation (0.1).

The purpose of this paper is to determine the class of all 1-dimensional diffusion processes having the representation (0.1). Incidentally, this class of diffusions is also characterized by the same kind of conditions as "normality" in [4]. We will see that this problem is closely related to a comparison theorem of Green functions of one-sided diffusion processes, which itself is of interest and has been studied by many authors (cf. [9]-[12]). In fact, characterizing this class in terms of speed measures will turn out to be equivalent to finding a necessary and sufficient condition for the Green functions of any two one-sided diffusion processes to have the same asymptotic behavior when $\alpha \rightarrow 0$ : Let $X(t)$ be a diffusion process with state space $(-\infty, \infty)$ having the infinitesimal generator $\frac{d}{d m} \frac{d}{d x}$ and $X_{1}(t)$ [or $X_{2}(t)$ ] the diffusion process with generator $\frac{d}{d m} \frac{d}{d x}$ restricted to $(-\infty, 0]$ [or $[0, \infty)$ resp.] (0 is a reflecting barrier). Then, denoting by $g_{\alpha}(x, y)$ [or $\mathrm{g}_{\alpha}{ }^{(i)}(x, y)$ ] the Green kernel of $X(t)$ [or $X_{i}(t)$, 
$i=1,2$, resp.] we will see that $\mathrm{g}_{\alpha}(x, y)$ has the representation $(0.1)$ if and only if

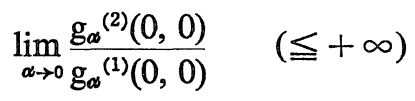

exists (Theorem 1).

Next we show that the latter holds if and only if

$$
\lim _{x \rightarrow \infty} \frac{u_{2}(x)}{u_{1}(x)} \quad(\leqq+\infty)
$$

exists, where $u_{1}(x)$ [or $u_{2}(x)$ ] denotes the inverse function of $x m(-x, 0)$ [or $x m[0, x)$ resp.] (Theorem 2). We will also obtain the explicit formula of $u(x, y)$ for processes having the representation $(0.1)$; if the process is nullrecurrent and if we take the scale $s(x) \equiv x$ as usual, we have

$$
u(x, y)=-\frac{1}{2}|x-y|+\theta(x+y),
$$

where $\theta$ is a constant $\left(|\theta| \leqq \frac{1}{2}\right)$ depending on the limit (0.4) .

In the last section we will give a Tauberian theorem for $h(\alpha)$, which is a generalization of the result for one sided-diffusions obtained by Y. Kasahara [9].

\section{§1. 1-Dimensional Generalized Diffusion Processes}

Let $m(d x)$ be a Borel measure on $\bar{R}=[-\infty, \infty]$ satisfying the following conditions: There exists an interval $\left[l_{-}, l_{+}\right] \subseteq \bar{R}$ such that

$$
m\left(\left(x_{-}, x_{+}\right)\right)<\infty \text { for every }\left(x_{-}, x_{+}\right) \subset\left[l_{-}, l_{+}\right],
$$

and

$$
m(\{x\})=\infty \text { for every } x \notin\left(l_{-}, l_{+}\right)
$$

We shall call a measure $m(d x)$ with the above property an inextensible measure. Without loss of generality we may assume $0 \in\left[l_{-}, l_{+}\right]$. Throughout this paper we use the convention:

$$
c \cdot \infty=\infty \quad c>0 \text { and }=0 \text { if } c=0, \text { and } \frac{1}{\infty}=0 .
$$

We introduce a generalized diffusion process $\left\{X_{t}\right\}$ with speed measure $m(d x)$ and scale $s(x) \equiv x$ as follows.

First define a pair of functions $\{\phi(x, \alpha), \psi(x, \alpha)\}$ on $R^{2}$ by the equations: 


$$
\begin{aligned}
& \phi(x, \alpha)=1+\alpha \int_{0}^{x}(x-y) \phi(y, \alpha) m(d y),{ }^{1)} \\
& \psi(x, \alpha)=x+\alpha \int_{0}^{x}(x-y) \psi(y, \alpha) m(d y) .
\end{aligned}
$$

Here for $x \notin\left(l_{-}, l_{+}\right)$we define

$$
\phi(x, \alpha)=|\psi(x, \alpha)|=\infty .
$$

$\{\phi, \psi\}$ are, roughly speaking, the unique solutions of

$$
\begin{aligned}
& \frac{d}{m(d x)} \frac{d \phi}{d x}=\alpha \phi, \quad \phi(0, \alpha)=1, \quad \phi^{\prime}(0-, \alpha)=0 \\
& \frac{d}{m(d x)} \frac{d \psi}{d x}=\alpha \psi, \quad \psi(0, \alpha)=0, \quad \psi^{\prime}(0-, \alpha)=1 .
\end{aligned}
$$

For $\alpha>0$, set

$$
h_{+}(\alpha)=\lim _{x \uparrow l_{+}} \frac{\psi(x, \alpha)}{\phi(x, \alpha)} \quad \text { and } \quad h_{-}(\alpha)=-\lim _{x \downarrow l_{-}} \frac{\psi(x, \alpha)}{\phi(x, \alpha)}
$$

Then $h_{ \pm}(\alpha)$ are non-decreasing analytic functions with the following integral representations:

$$
h_{ \pm}(\alpha)=\int_{R_{ \pm}} \frac{d x}{\phi(x, \alpha)^{2}},
$$

where $R_{+}=[0, \infty)$ and $R_{-}=(-\infty, 0]$.

Let

$$
u_{ \pm}(x, \alpha)=\phi(x, \alpha) \mp \frac{1}{h_{ \pm}(\alpha)} \psi(x, \alpha)
$$

and

$$
\frac{1}{h(\alpha)}=\frac{1}{h_{+}(\alpha)}+\frac{1}{h_{-}(\alpha)}
$$

Then, for $\alpha>0, u_{+}(x, \alpha)\left[u_{-}(x, \alpha)\right]$ is a non-increasing [non-decreasing, resp.] and non-negative function. Since their Wronskian equals $h(\alpha)^{-1}$, we can define the Green function $g_{\alpha}(x, y)$ of order $\alpha$ as follows:

$$
g_{\alpha}(x, y)=g_{\alpha}(y, x)=h(\alpha) u_{+}(x, \alpha) u_{-}(y, \alpha) \quad \text { for } \quad x \geqq y .
$$

Let $S$ be the support of the measure $m(d x)$ on $\left(l_{-}, l_{+}\right)$and $B(S)$ be the 1) $\int_{x}^{y}=\int_{[y \cdot x)}$ for $y<x$, and $=-\int_{[x \cdot y)}$ for $x<y$. 
space of all bounded measurable functions on $S$. Define the Green operator $G_{a}$ on $B(S)$ by

$$
G_{\infty} f(x)=\int_{s} g_{a}(x, y) f(y) m(d y)
$$

Then, unless $S$ is empty, we can use stochastic time change to construct a unique time homogeneous strong Markov process $X=\left\{X_{t}, \zeta\right\}$ on $S$ satisfying

$$
G_{a} f(x)=E_{x}\left(\int_{0}^{\zeta} e^{-\infty t} f\left(X_{t}\right) d t\right)
$$

for every $f \in B(S)$ and $\alpha>0$. We call this process $X$ a generalized diffusion process with speed measure $m(d x)$ and scale $s(x) \equiv x$. Let $a_{+}\left[a_{-}\right]$be the supremum [the infimum resp.] of $S$. If $a_{ \pm}$and $m\left(\left(a_{-}, a_{+}\right)\right)$are finite, then $\phi(x)=$ $G_{o f} f(x)(f \in B(s), \alpha>0)$ satisfies the boundary conditions:

$$
\phi\left(a_{ \pm}\right)+\left(l_{ \pm}-a_{ \pm}\right) \phi^{\prime}\left(a_{ \pm}\right)=0
$$

(in case $l_{+}=\infty\left[l_{-}=-\infty\right]$, the above equality must be interpreted as $\phi^{\prime}\left(a_{+}\right)=0$ $\left[\phi^{\prime}\left(a_{-}\right)=0\right.$ resp.]). Our diffusion process turns out to be an ordinary one if and only if $S$ is an interval.

\section{§2. An Asymptotic Expression of $g_{\alpha}(x, y)$}

First note

$$
l_{+}=h_{+}(0+) \text { and } l_{-}=-h_{-}(0+) .
$$

This is easily seen from (1.2), and this fact will be frequently used later. Moreover the process is recurrent if and only if $h(0+)=\infty$, which is equivalent to $l_{+}=-l_{-}=\infty$ (cf. [9]). Let $\dot{S}=S \cap\left(a_{-}, a_{+}\right)$and define

$$
C_{0}(\stackrel{\circ}{S})=\left\{\phi ; \phi \text { is a continuous function with compact support in } S^{\circ}\right\} \text {. }
$$

Set

$$
\sigma(x)=\int_{0}^{x}(x-y) m(d y), \quad \tau(x)=\int_{0}^{x}(x-y) y m(d y)
$$

and

$$
l=h_{+}(0+)+h_{-}(0+)=l_{+}-l_{-} .
$$

Then our first theorem is

Theorem $\mathrm{H}_{\text {. Suppose }} \$ \neq \emptyset$. Then the following four statements are equivalent: 
(i) $g_{\alpha}(x, y)$ admits the following expression with a constant $\theta\left(|\theta| \leqq \frac{1}{2}\right)$

$$
\begin{aligned}
g_{\omega}(x, y)= & g_{\omega}(0,0)-\frac{1}{2}|x-y|+\theta(x+y)+\frac{1}{m(R)}\{\sigma(x)+\sigma(y)\} \\
& -\frac{1}{l} x y+\varepsilon(x, y ; \alpha),
\end{aligned}
$$

where $\varepsilon(x, y ; \alpha)$ converges to zero as $\alpha \rightarrow 0$ uniformly in $(x, y)$ on every compact set of $\dot{S} \times \dot{S}$.

(ii) The following limit exists;

$$
\lim _{\alpha \rightarrow 0} \frac{h_{-}(\alpha)}{h_{+}(\alpha)}=\hat{\theta}, \quad 0 \leqq \hat{\theta} \leqq \infty .
$$

(iii) For an $f \in C_{0}(\stackrel{\circ}{S})$ with $\int f(x) m(d x)=0$ and $\int x f(x) m(d x) \neq 0$, the function $G_{a} f(x)$ converges to some function as $\alpha \rightarrow 0$ at every point $x \in \dot{S}$.

(iv) For every $f \in C_{0}(\stackrel{\circ}{S})$ with $\int f(x) m(d x)=0, G_{a} f(x)$ converges to $G_{0} f(x)$ uniformly on every compact set of $\dot{S}$.

Here we have the relations;

$$
\hat{\theta}=\frac{1-2 \theta}{1+2 \theta} \quad \text { and } \quad g_{a}(0,0)=h(\alpha) .
$$

Furthermore, the limit function in (iv) is given by

$$
G_{0} f(x)=\int_{s}\left\{-\frac{1}{2}|x-y|+\theta y+\frac{\sigma(y)}{m(R)}-\frac{1}{l} x y\right\} f(y) m(d y) .
$$

Remark 1. Since $h_{+}(\alpha) \equiv \infty$ if and only if $m\left(R_{+}\right)=0$, if at least one of the boundary points of the state $S$ is regular the condition (ii) is satisfied automatically.

Remark 2. If the process is transient, which is equivalent to $h(0+)<\infty$, for every $f \in C_{0}^{\circ}(S) \quad G_{a} f(x)$ converges to $G_{0} f(x)$ uniformly on every compact set of $S$, where

$$
G_{0} f(x)=\int_{s}\left\{h(0+)-\frac{1}{2}|x-y|+\theta(x+y)-\frac{1}{l} x y\right\} f(y) m(d y) .
$$

We remark that $h(0+)$ can be written as

$$
h(0+)=-\frac{l_{+} l_{-}}{l_{+}-l_{-}} .
$$


In this case the term $\sigma(y) / m(R)$ will automatically disappear in the expression $G_{0}$, because $h(0+)<\infty$ implies $m(R)=\infty$.

Remark 3. We can also prove that $\sup _{\substack{0<\infty<1 \\ x \in S}}\left|G_{\infty} f(x)\right|<\infty$ in (iv).

To prove the theorem, we need two lemmas.

Lemma 2.1. The following asymptotic expression holds:

$$
\alpha h(\alpha)=\frac{1}{m(R)}+o(1) \quad \text { as } \quad \alpha \rightarrow 0
$$

Lemma 2.2. Suppose $S \neq \emptyset$. Let $\hat{\theta}(\alpha)=\frac{h_{-}(\alpha)}{h_{+}(\alpha)}$. Then $g_{\alpha}(x, y)$ can be expressed as follows:

$$
\begin{aligned}
g_{a}(x, y)= & g_{a}(0,0)-\frac{1}{2}|x-y|-\frac{1}{l} x y+\frac{1}{m(R)}\{\sigma(x)+\sigma(y)\} \\
& +\frac{1}{2} \frac{1-\hat{\theta}(\alpha)}{1+\hat{\theta}(\alpha)}(x+y)+\varepsilon(x, y ; \alpha)
\end{aligned}
$$

where $\varepsilon(x, y ; \alpha)$ converges to zero as $\alpha \rightarrow 0$ uniformly in $(x, y)$ on every compact subset of $\dot{S} \times \stackrel{\circ}{S}$.

Proof of Lemma 2.1. Since we have

$$
\frac{1}{\alpha h(\alpha)}=\frac{1}{\alpha h_{+}(\alpha)}+\frac{1}{\alpha h_{-}(\alpha)} \quad(\text { see }(1.3))
$$

we need only to show

$$
\alpha h_{+}(\alpha)=\frac{1}{m\left(R_{+}\right)}+o(1) \quad \text { as } \quad \alpha \rightarrow 0 .
$$

In case $h_{+}(\alpha) \equiv \infty$ (i.e. $m\left(R_{+}\right)=0$ ), (2.3) is valid. If otherwise, by (1.2) we have

$$
\alpha h_{+}(\alpha)=\int_{0}^{\infty} \frac{d x}{\phi\left(\frac{x}{\alpha}, \alpha\right)^{2}} .
$$

By a simple change of variables, we see

$$
\phi\left(\frac{x}{\alpha}, \alpha\right)=1+\int_{0}^{x}(x-y) \phi\left(\frac{y}{\alpha}, \alpha\right) m\left(\frac{d y}{\alpha}\right),
$$

from which it follows that $\phi\left(\frac{x}{\alpha}, \alpha\right) \rightarrow 1+m\left(R_{+}\right) x$ as $\alpha \rightarrow 0$ and that 


$$
\begin{aligned}
\phi\left(\frac{x}{\alpha}, \alpha\right) & \geqq 1+\int_{0}^{x}(x-y) m\left(\frac{d y}{\alpha}\right) \\
& \geqq 1+\frac{x}{2} m\left(\left[0, \frac{x}{2 \alpha}\right)\right) .
\end{aligned}
$$

Hence, by the dominated convergence theorem, we have

$$
\alpha h_{+}(\alpha) \rightarrow \int_{0}^{\infty} \frac{d x}{\left\{1+m\left(R_{+}\right) x\right\}^{2}}=\frac{1}{m\left(R_{+}\right)},
$$

which completes the proof.

Proof of Lemma 2.2. Let

$$
\begin{aligned}
\phi_{k}(x) & =\int_{0}^{x}(x-y) \phi_{k-1}(y) m(d y) \quad(k \geqq 1) \\
\phi_{0}(x) & =1 .
\end{aligned}
$$

Then the solution of (1.1) can be represented as

$$
\phi(x, \alpha)=\sum_{k=0}^{\infty} \alpha^{k} \phi_{k}(x)
$$

which implies

$$
\phi(x, \alpha)=1+\alpha \sigma(x)+O\left(\alpha^{2}\right) \quad \text { as } \quad \alpha \rightarrow 0,
$$

uniformly on every compact set of $\dot{S}$. Similarly we have

$$
\psi(x, \alpha)=x+\alpha \tau(x)+O\left(\alpha^{2}\right) \quad \text { as } \quad \alpha \rightarrow 0 .
$$

Therefore observing $h_{ \pm}(\alpha)^{-1}$ are bounded, we see

$$
\begin{aligned}
u_{ \pm}(x, \alpha) & \equiv \phi(x, \alpha) \mp \frac{1}{h_{ \pm}(\alpha)} \psi(x, \alpha) \\
& =1 \mp \frac{x}{h_{ \pm}(\alpha)}+\alpha \sigma(x) \mp \frac{\alpha}{h_{ \pm}(\alpha)} \tau(x)+O\left(\alpha^{2}\right) .
\end{aligned}
$$

The assumption $S \neq \emptyset$ implies $h(\alpha)<\infty$, hence

$$
0 \leqq \frac{h(\alpha)}{h_{ \pm}(\alpha)} \leqq 1 \text { and } \frac{h(\alpha)}{h_{+}(\alpha) h_{-}(\alpha)}=\frac{1}{h_{+}(\alpha)+h_{-}(\alpha)}
$$

Consequently, applying (2.1), we have for $x \geqq y$

$$
\begin{aligned}
g_{\alpha}(x, y) & =h(\alpha) u_{+}(x, \alpha) u_{-}(y, \alpha) \\
& =h(\alpha)-\frac{h(\alpha)}{h_{+}(\alpha)} x+\frac{h(\alpha)}{h_{-}(\alpha)} y+\alpha h(\alpha)\{\sigma(x)+\sigma(y)\}
\end{aligned}
$$




$$
\begin{aligned}
& -\frac{1}{h_{+}(\alpha)+h_{-}(\alpha)} x y+O(\alpha) \\
= & g_{\alpha}(0,0)-\frac{1}{2}|x-y|-\frac{1}{l} x y+\frac{1}{m(R)}\{\sigma(x)+\sigma(y)\} \\
& +\frac{1}{2} \frac{1-\hat{\theta}(\alpha)}{1+\hat{\theta}(\alpha)}(x+y)+o(1) \quad \text { as } \quad \alpha \rightarrow 0,
\end{aligned}
$$

which completes the proof.

Proof of Theorem 1. We can obtain the theorem immediately! from the expression (2.2).

Q.E.D.

We call a generalized diffusion process normal if it satisfies the condition (iv) of Theorem 1 (and hence if it satisfies anyone of the conditions in Thorem 1.). Thus, if $X$ is normal there corresponds a constant $\theta\left(|\theta| \leqq \frac{1}{2}\right)$ such that (i) of Theorem 1 holds. We call then that $X$ is normal with parameter $\theta$. The concept of normality for recurrent processes was originally introduced by Kemeny-Snell in the case of Markov chain and our definition is similar to that of R. Kondo [4].

\section{§3. A Condition for the Process to Be Normal}

It is of interest to give conditions for the process to be normal in terms of the speed measure $m(d x)$. By virtue of (ii) of Theorem 1, this problem can be reduced to the following: Under what conditions on two inextensible measures on $[0, \infty] m_{1}(d x)$ and $m_{2}(d x)$ do their Green functions have the same asymptotic behavior as $\alpha \rightarrow 0$ ? Our aim here is to find a necessary and sufficient condition.

Every inextensible measure $m(d x)$ on $[0, \infty]$ can be identified with a nondecreasing, right-continuous function $m(x)=m([0, x]), 0 \leqq x<\infty$. We will denote the class of all such functions by $\mathscr{M}$. For every $m \in \mathscr{M}$, using the solution (1.1), we define

$$
h(\alpha)=\int_{0}^{\infty} \frac{d x}{\phi(x, \alpha)^{2}} .
$$

For simplicity, we call this function $h(\alpha)$ the $h$-function of $m$. It should be noted that $h(\alpha) \equiv 0$ if $m(x) \equiv \infty$ and $h(\alpha) \equiv \infty$ if $m(x) \equiv 0$. Let $\mathcal{A}$ be the set of all functions $h(\alpha)$ such that

$$
\begin{aligned}
& h(\alpha) \text { is holomorphic on } \mathbb{C} \backslash(-\infty, 0], \\
& (\operatorname{Im} \alpha)(\operatorname{Im} h(\alpha)) \leqq 0 \text { for every } \alpha \in \mathbb{C} \backslash \mathbb{R},
\end{aligned}
$$




$$
\begin{aligned}
& \text { and } h(\alpha) \geqq 0 \text { for every } \alpha \in(0, \infty) \\
& \text { or } h(\alpha) \equiv \infty .
\end{aligned}
$$

Then (3.1) gives a one-to-one correspondence between $\mathscr{M}$ and $\mathscr{H}$ (M. G. Krein [13]). We next define

$$
u(x)=\sup \{y ; y m(y) \leqq x\}
$$

and call it the $u$-function of $m$. Remark that $u(x)$ is a non-decreasing continuous function tending to $\infty$ as $x \rightarrow \infty$ if $u(x) \neq \equiv$, or equivalently, if $m(x) \neq 0$. To study the asymptotic behavior of $h(\alpha), u$-function is more convenient than $m(x)$ itself not only because $u$-functions are continuous but also because we can prove that

$$
\frac{1}{2} \leqq \frac{h(\alpha)}{u\left(\frac{1}{\alpha}\right)} \leqq 2
$$

holds for every $\alpha>0$ provided $u \neq \equiv$. However, we do not go into details of (3.2) since we will not use (3.2) itself in this paper. We need only the following two lemmas:

Lemma 3.1. If $h(\alpha) \in \mathcal{H}$ corresponds to $u(x)$, then for every positive constants $a$ and $b, a h(b \alpha)$ corresponds to $a u(x / b)$.

Proof. The assertion can be easily proved by a simple change of variables in (1.1) and (3.1).

Q.E.D

Lemma 3.2. Let $u_{n}$ and $h_{n}$ be the $u$-functions and $h$-functions of $m_{n}(x) \in \mathscr{M}$ $(n=1,2, \cdots)$ respectively. Then

$$
\lim _{n \rightarrow \infty} u_{n}(x)=u_{0}(x) \quad \text { for every } \quad x>0
$$

if and only if

$$
\lim _{n \rightarrow \infty} h_{n}(\alpha)=h_{0}(\alpha) \quad \text { for every } \quad \alpha>0 \text {. }
$$

Proof. It is easy to see that (3.3) is equivalent to

$$
\lim _{n \rightarrow \infty} m_{n}(x)=m_{0}(x)
$$

at every continuity point of $m_{0}(x)$. On the other hand, (3.5) is equivalent to (3.4) (see Theorem 1 in [9]).

Theorem 2. Let $h_{i}$ and $u_{i}(i=1,2)$ be the h-functions and u-functions of $m_{i} \in \mathcal{M}$. Then 


$$
\lim _{\substack{\alpha \rightarrow 0 \\[\infty]}} \frac{h_{2}(\alpha)}{h_{1}(\alpha)}=\lim _{\substack{x \rightarrow \infty \\ \text { ro] }}} \frac{u_{2}(x)}{u_{1}(x)} \quad(\leqq \infty)
$$

as far as at least one side of (3.6) exists.

Proof. We first remark that, from any sequence $\left\{m_{n}(x)\right\}_{n=1}^{\infty} \subset \mathscr{M}$, we can choose a subsequence $\left\{m_{n},(x)\right\}$ and $m^{*}(x) \in \mathscr{M}$ such that

$$
\lim _{n^{\prime} \rightarrow \infty} m_{n^{\prime}}(x)=m^{*}(x)
$$

holds at every continuity point of $m^{*}(x)$. Therefore, denoting by $u_{n}(x)$ and $u^{*}(x)$ the $u$-functions of $m_{n}(x)$ and $m^{*}(x)$ respectively, we see

$$
\lim _{n \rightarrow \infty} u_{n}(x)=u^{*}(x), \quad x>0 .
$$

From this it easily follows that for any sequence $\left\{a_{n}\right\}_{n=1}^{\infty}$ of positive numbers there exists a subsequence $\left\{b_{n}\right\}$ of $\left\{a_{n}\right\}$ such that

$$
\lim _{n \rightarrow \infty} \frac{u_{1}\left(b_{n} x\right)}{u_{1}\left(b_{n}\right)}=u^{*}(x), \quad x>0,
$$

where $u^{*}(x)$ is the $u$-function of some $m^{*}(x) \in \mathscr{M}$. However, by Lemmas 3.1 and 3.2, (3.7) is equivalent to

$$
\lim _{n \rightarrow \infty} \frac{1}{u_{1}\left(b_{n}\right)} h_{1}\left(\frac{\alpha}{b_{n}}\right)=h^{*}(\alpha),
$$

where $h^{*}(\alpha)$ is the $h$-function of $m^{*}(x)$. Now assume

$$
\lim _{\substack{n \rightarrow \infty \\[\infty 1}} \frac{h_{2}(\alpha)}{h_{1}(\alpha)}=\theta \quad(0 \leqq \theta \leqq \infty) .
$$

Then, if $a_{n} \rightarrow \infty$ [or 0], from (3.7)' it follows

$$
\lim _{n \rightarrow \infty} \frac{1}{u_{1}\left(b_{n}\right)} h_{2}\left(\frac{\alpha}{b_{n}}\right)=\theta h^{*}(\alpha) .
$$

Using Lemmas 3.1 and 3.2 again, we see that (3.9) is equivalent to

$$
\lim _{n \rightarrow \infty} \frac{u_{2}\left(b_{n} x\right)}{u_{1}\left(b_{n}\right)}=\theta u^{*}(x) .
$$

Setting $x=1$, we have

$$
\lim _{n \rightarrow \infty} \frac{u_{2}\left(b_{n}\right)}{u_{1}\left(b_{n}\right)}=\theta
$$


Since $\left\{a_{n}\right\}$ is arbitrary, (3.11) implies

$$
\lim _{\substack{n \rightarrow \infty \\ \text { [0] }}} \frac{u_{2}(x)}{u_{1}(x)}=\theta .
$$

We can prove the converse similary, so we omit the proof.

Q.E.D.

As an easy corollary of the theorem above we have the following theorem which answers the question we stated in the head of this section.

Theorem 2'. Let $X$ be a generalized diffusion with speed measure $m(d x)$. Then $X$ is normal with parameter $\theta$ if and only if

$$
\lim _{n \rightarrow \infty} \frac{u_{-}(x)}{u_{+}(x)}=\hat{\theta} \quad\left(2 \theta=\frac{1-\hat{\theta}}{1+\hat{\theta}}\right),
$$

where $u_{+}$and $u_{-}$are the inverse to $x m([0, x])$ and to $x m([-x, 0])$, respectively.

Remark. It is not difficult to see that

$$
\lim _{n \rightarrow \infty} \frac{m([-x, 0])}{m([0, x])}=1
$$

implies (3.13) with $\hat{\theta}=1$. However, the converse is not necessarily true. For example, set $m([-x, 0])=x e^{x}$ and $m([0, x])=e^{x}$. Then, (3.14) fails to hold though (3.13) with $\hat{\theta}=1$ is valid. In fact, (3.13) is equivalent to the following:

$$
\lim _{n \rightarrow \infty} \inf \frac{m([-\lambda x, 0])}{m([0, x])} \geqq \frac{1}{\hat{\theta}}, \quad \text { for } \quad \lambda>\hat{\theta},
$$

and

$$
\lim _{n+\infty} \sup \frac{m([-\lambda x, 0])}{m([0, x])} \leqq \frac{1}{\hat{\theta}}, \quad \text { for } \quad 0<\lambda<\hat{\theta}
$$

\section{\$4. A Condition for $h(\alpha)=g_{\alpha}(0,0)$ to Be Regularly Varying}

One of the authors [8] showed the following limit theorem for normal processes. Let $X=\left\{X_{t}\right\}$ be a recurrent normal process and let $f \in C_{0}(S)$ with $\int f(x) m(d x)=0$. For some non-negative increasing function $u(t)$ we consider the limiting distributions of random variables

$$
\frac{1}{u(t)} \int_{0}^{t} f\left(X_{s}\right) d s, \quad t \rightarrow \infty
$$

If $h(\alpha)$ varies regularly at 0 , then (4.1) converges in law to the bilateral Mittag- 
Leffler distribution for $u(t)=h(1 / t)$. Conversely, if (4.1) converges in law to some non-degenerate distribution with some $u(t)$, then $h(\alpha)$ varies regularly at 0 . Therefore it is important to give a condition in terms of the speed measure $m(d x)$ for $h(\alpha)$ to be regularly varying. In case $m\left(R_{-}\right)=0$, the following lemma was proved in [9].

Lemma 4.1. Let $m \in \mathscr{M}$. Then

$$
\lim _{x \rightarrow 0} \frac{m(\lambda x)}{m(x)}=\lambda^{\frac{1}{\beta}-1} \quad \text { for } \quad \lambda>0
$$

(equivalently $\lim _{x \rightarrow \infty} \frac{u(\lambda x)}{u(x)}=\lambda^{\beta}$ ) if and only if

$$
\lim _{\alpha \rightarrow 0} \frac{h(\lambda \alpha)}{h(\alpha)}=\lambda^{-\beta} \quad \text { for } \quad \lambda>0,
$$

where $0 \leqq \beta \leqq 1$ and $\lambda^{\circ}$ means $\infty$ for $\lambda>1$ and 0 for $0<\lambda<1$.

In case $m\left(R_{-}\right)>0$, if the process is normal, we can show the following

Theorem 3. Suppose the process $X$ corresponding to $m$ is normal with parameter $\theta\left(|\theta| \leqq \frac{1}{2}\right)$. Then for some $0 \leqq \beta \leqq 1$ we have

$$
\lim _{\alpha \rightarrow 0} \frac{h(\lambda \alpha)}{h(\alpha)}=\lambda^{-\beta} \quad \text { for } \quad \lambda>0
$$

if and only if

$$
\lim _{x \rightarrow \infty} \frac{u(\lambda x)}{u(x)}=\lambda^{\beta} \quad \text { for } \quad \lambda>0
$$

holds, where $u(x)=\min \left\{u_{+}(x), u_{-}(x)\right\}$.

Proof. First consider the case $0 \leqq \theta \leqq \frac{1}{2}$ and hence $0 \leqq \hat{\theta} \leqq 1$. Assume (4.2) holds. From Theorem $2^{\prime}$ it follows

$$
\lim _{\alpha \rightarrow 0} \frac{h_{-}(\alpha)}{h_{+}(\alpha)}=\lim _{x \rightarrow \infty} \frac{u_{-}(x)}{u_{+}(x)}=\hat{\theta} .
$$

This together with (1.3) implies

$$
\lim _{\alpha \rightarrow 0} \frac{h_{-}(\alpha)}{h(\alpha)}=1+\lim _{\alpha \rightarrow 0} \frac{h_{-}(\alpha)}{h_{+}(\alpha)}=1+\hat{\theta}
$$

Therefore by (4.2) we have 


$$
\lim _{\alpha \rightarrow 0} \frac{h_{-}(\lambda \alpha)}{h_{-}(\alpha)}=\lambda^{-\beta}, \quad \lambda>0
$$

which implies by Lemma 4.1

$$
\lim _{x \rightarrow \infty} \frac{u_{-}(\lambda x)}{u_{-}(x)}=\lambda^{\beta}
$$

Noting $0 \leqq \hat{\theta} \leqq 1$, we have by (4.4)

$$
\lim _{x \rightarrow \infty} \frac{u(\lambda x)}{u(x)}=\lambda^{\beta}
$$

We can discuss the case $-\frac{1}{2} \leqq \theta<0$ similarly as above. Since it is easy to reverse the above argument, we can obtain the theorem. Q.E.D.

\section{References}

[1] Hunt, G., Markov processes and potentials, Illinois J. Math., 1 (1957), 44-93, 316-369, 2 (1958), 151-213.

[2] Takeuchi, J., Yamada, T. and Watanabe, S., Stable processes, Seminar on Prob., 13 (1962) (in Japanese).

[3] Kemeny-J. G. nad Snell, L., Potentials for denumerable Markov chains, J. Math. Anal. Appl., 3 (1961), 196-260.

[4] Kondo, R., On weak potential operators for recurrent Markov processes, J. Math. Kyoto Univ., II (1971), 11-41.

[5] Yosida, K., On the potential operators associated with Brownian motions, J. Analyse Math., 23 (1970), 461-465.

[6] Sato, K., Potential operators for Markov processes, Proc. 6th Berkeley Symp. Math. Stat. Prob. Univ. Calif., 3 (1971), 193-211.

[7] Arakawa, T. and Takeuchi, J., On the potential operators associated with Bessel processes, Z. Wahrsch. und Verw. Geb., 40 (1977), 83-90.

[8] Kasahara, Y., Limit theorems of occupation times for Markov processes, Publ. RIMS Kyoto Univ., 12 (1977), 801-818.

[9] Kasahara, Y., Spectral theory of generalized second order differential operators and its applications to Markov processes, Japan J. Math., 1 (1975), 67-84.

[10] Kac, I. S., Generalization of an asymptotic formula of V. A. Marcenko for spectral functions of a second-order boundary value problem, Izv. Akad. Nauk. SSSR ser. Mat., 37 (1973), $422-436$ (in Russian); Math. USSR Izv., 7 (1973), 422-436 (Engl. Transl.).

[11] Ikeda, N., Kotani, S. and Watanabe, S., On the asymptotic behaviors of transition densities in generalized diffusion processes, Seminar on Probab., 41 (1975), 104-120 (in Japanese).

[12] Tomisaki, M., Comparison theorems in generalized diffusion processes, Mem. Fac. Sci. Kyushu Univ., 30 (1976), 247-256.

[13] Krein, M. G.. On a generalization of investigations of Stieltjes, Dokl. Akad. Nauk SSSR, 87 (1952), 881-884. 